H. Nomoto

Nagoya Math. J.

Vol. 59 (1975), 1-8

\title{
SQUARE OF BROWNIAN MOTION
}

\section{To Professor Kiyoshi Ito on the occasion of his 60th birthday}

\author{
HISAO NOMOTO
}

\section{Introduction.}

Let $X_{t}$ be a stochastic process and $Y_{t}$ be its square process. The present note is concerned with the solution of the equation $X_{t}^{2}=Y_{t}$ assuming $Y_{t}$ is given. In [4], F. A. Grünbaum proved that certain statistics of $Y_{t}$ are enough to determine those of $X_{t}$ when it is a centered, nonvanishing, Gaussian process with continuous correlation function. In connection with this result, we are interested in sample function-wise inference, though it is far from generalities. A glance of the equation $X_{t}^{2}=Y_{t}$ shows that the difficulty is related how to pick up a sign of $\pm \sqrt{Y_{t}}$. Thus if we know that $X_{t}$ has nice sample process such as the zero crossings are finite, no tangencies, in any finite time interval, then observations of these statistics will make it sure to find out sample functions of $X_{t}$ from those of $Y_{t}$ (see [2]). The purpose of this note is to consider the above problem from this point of view.

In section 2, we shall construct a new standard random walk as an explicit functional of the square process $S_{n}^{2}$ of the given standard random walk $S_{n}$ availing of sample functions properties, reflection at $x=0$, of $\sqrt{S_{n}^{2}}$. In section 3 , we shall consider the same problem to the previous section for Brownian motion. In this case, we make use of the local time at $x=0$ of reflecting Brownian motion on $[0, \infty)$ to get a new Brownian motion from an "inverse map" of the map $B_{t} \rightarrow B_{t}^{2}$.

As to the terminology about Markov processes we refer to [1] and [6].

\section{Random walk.}

Before to state theorems, we prepare two lemmas.

Received November 29, 1974. 
LEMMA 1. Let $\left[X_{n} ; n \geqq 0\right]$ be a Markov chain on all nonnegative integers $\{0,1,2, \cdots\}$ with the transition probability $p_{00}=p_{01}=\frac{1}{2}$ and $p_{i, i \pm 1}=\frac{1}{2}(i \geqq 1)$. Then $u\left(X_{n}-X_{n-1}\right) \quad(n \geqq 1)$ are independent with common distribution $P_{0}\left\{u\left(X_{n}-X_{n-1}\right)= \pm 1\right\}=\frac{1}{2}$. Where $u(x)=x$ or $u(x)=-1$ according as $x= \pm 1$ or $x=0$.

Proof. $1^{\circ}$. Since

$$
P_{i}\left\{u\left(X_{1}-X_{0}\right)=a\right\}=\frac{1}{2} \quad \text { for } a= \pm 1 \text { and } i \geqq 0,
$$

we see

$$
\begin{aligned}
P_{0}\left\{u\left(X_{n}-X_{n-1}\right)=a\right\} & =E_{0}\left[P_{X_{n-1}}\left\{u\left(X_{1}-X_{0}\right)=a\right\}\right] \\
& =\sum_{k \geq 0} p_{0 k}^{(n-1)} P_{k}\left\{u\left(X_{1}-X_{0}\right)=a\right\} \\
& =\frac{1}{2} \sum_{k \geq 0} p_{0 k}^{(n-1)}=\frac{1}{2} \cdot{ }^{1)}
\end{aligned}
$$

That is, each $u\left(X_{n}-X_{n-1}\right)$ has the same distribution with the fair cointossing game.

Next we shall prove that the system $\left[u\left(X_{n}-X_{n-1}\right) ; n \geqq 1\right]$ is independent with respect to $P_{0}$.

$2^{\circ}$. First we compute the probability

$$
p_{n}=P_{1}\left\{u\left(X_{1}-X_{0}\right)=a_{1}, \cdots, u\left(X_{n}-X_{n-1}\right)=a_{n}\right\}, \quad\left(a_{j}= \pm 1\right) .
$$

Let $\tau=\operatorname{Min}\left\{k \geqq 1 \mid X_{k}=0\right\}$ be the hitting time to $x=0$. Since $P_{k}\{\tau<\infty\}=1$, we have

$$
p_{n}=\sum_{\ell=1}^{\infty} P_{1}\left\{u\left(X_{1}-X_{0}\right)=a_{1}, \cdots, u\left(X_{n}-X_{n-1}\right)=a_{n}, \tau=\ell\right\} .
$$

For given $a_{j}$ 's, consider the following two cases;

Case 1. There exists a $k$ such that

$$
\begin{aligned}
& 1 \leqq k \leqq n, 1+a_{1}+\cdots+a_{j} \geqq 1, \\
& \quad(j=1, \cdots, k-1) \text { and } 1+a_{1}+\cdots+a_{k}=0,
\end{aligned}
$$

and

Case 2. $1+a_{1}+\cdots+a_{j} \geqq 1,(j=1,2, \cdots, n)$.

In the case 1 , using the strong Markov property, we have

\footnotetext{
1) $p_{i j}^{(n)}$ denotes the transition probability in $n$ steps.
} 


$$
\begin{aligned}
p_{n} & =P_{1}\left\{u\left(X_{1}-X_{0}\right)=a_{1}, \cdots, u\left(X_{n}-X_{n-1}\right)=a_{n}, \tau=k\right\} \\
& =\frac{1}{2} P_{1+a_{1}}\left\{u\left(X_{1}-X_{0}\right)=a_{2}, \cdots, u\left(X_{n-1}-X_{n-2}\right)=a_{n}, \tau=k-1\right\} \\
& =\cdots \\
& =\frac{1}{2^{k}} P_{1+a_{1}+\cdots+a_{k}}\left\{u\left(X_{1}-X_{0}\right)=a_{k+1}, \cdots, u\left(X_{n-k}-X_{n-k-1}\right)=a_{n}\right\},
\end{aligned}
$$

so that (4) implies

$$
\begin{aligned}
p_{n}=\frac{1}{2^{k}} P_{0}\left\{u\left(X_{1}-X_{0}\right)=a_{k+1}, \cdots, u\left(X_{n-k}-X_{n-k-1}\right)\right. & \left.=a_{n}\right\}, \\
& \text { with } X_{-1}=0 .
\end{aligned}
$$

In the case 2 , by the same computations as above, we get

$$
\begin{aligned}
p_{n} & =\sum_{\ell=n+1}^{\infty} P_{1}\left\{u\left(X_{1}-X_{0}\right)=a_{1}, \cdots, u\left(X_{n}-X_{n-1}\right)=a_{n}, \tau=\ell\right\} \\
& =\frac{1}{2^{n}} \sum_{\ell=n+1}^{\infty} P_{1+a_{1}+\cdots+a_{n}}\{\tau=\ell-n\} \\
& =\frac{1}{2^{n}} .
\end{aligned}
$$

$3^{\circ}$. Now we shall prove that the probability

$$
q_{n}=P_{0}\left\{u\left(X_{1}-X_{0}\right)=a_{1}, \cdots, u\left(X_{n}-X_{n-1}\right)=a_{n}\right\}
$$

is equal to $1 / 2^{n}$ by the induction for $n$ together with (5) and (6). Since $q_{1}=1$ by (1), assume that $q_{k}=1 / 2^{k}$ for any $k \leqq n-1$ and $\left\{a_{1}, \cdots, a_{k}\right\}$ with $a_{j}= \pm 1$. Then, it holds that

$$
\begin{aligned}
q_{n}= & E_{0}\left[P_{X_{1}}\left\{u\left(X_{1}-X_{0}\right)=a_{2}, \cdots, u\left(X_{n-1}-X_{n-2}\right)=a_{n}\right\} ; u\left(X_{1}-X_{0}\right)=a_{1}\right]^{2)} \\
= & P_{0}\left\{X_{1}=0, u\left(X_{1}-X_{0}\right)=a_{1}\right\} P_{0}\left\{u\left(X_{1}-X_{0}\right)=a_{2}, \cdots, u\left(X_{n-1}-X_{n-2}\right)=a_{n}\right\} \\
& +P_{0}\left\{X_{1}=1, u\left(X_{1}-X_{0}\right)=a_{1}\right\} P_{1}\left\{u\left(X_{1}-X_{0}\right)=a_{2}, \cdots, u\left(X_{n-1}-X_{n-2}\right)\right. \\
& \left.\quad=a_{n}\right\} .
\end{aligned}
$$

Therefore, by the assumption of the induction together with (1), (5) and (6), we get

$$
\begin{aligned}
q_{n} & =\frac{1}{2^{n-1}} P_{0}\left\{X_{1}=0, u\left(X_{1}-X_{0}\right)=a_{1}\right\}+\frac{1}{2^{n-1}} P_{0}\left\{X_{1}=1, u\left(X_{1}-X_{0}\right)=a_{1}\right\} \\
& =\frac{1}{2^{n-1}} P_{0}\left\{u\left(X_{1}-X_{0}\right)=a_{1}\right\}=\frac{1}{2^{n}} .
\end{aligned}
$$

2) $E_{x}[f ; A]=\int_{A} f(w) P_{x}(d w)$. 
That is

$$
q_{n}=\prod_{k=1}^{n} P_{0}\left\{u\left(X_{k}-X_{k-1}\right)=a_{k}\right\}
$$

This proves the lemma.

Consider a Markov chain on $\{0,1,2, \cdots\}$ determined by

$$
p_{01}=1 \text { and } p_{j, j \pm 1}=\frac{1}{2}(j \geqq 1)
$$

Let

$$
\begin{aligned}
A_{n} & =\sum_{k=1}^{n} \chi_{N}\left(X_{k}\right), \quad(n \geqq 1), \quad A_{0}=0,,^{3)} \\
A_{n}^{-1} & =\operatorname{Min}\left\{k \geqq 1 \mid A_{k} \geqq n\right\}, \quad A_{0}^{-1}=0
\end{aligned}
$$

be the additive functional of $X_{n}$ and its inverse respectively.

Then we have the following lemma.

LEMMA 2. The time changed process $\hat{X}_{n}=X\left(A_{n}^{-1}\right)$ is the Markov chain on $\{0,1,2, \cdots\}$ with the transition probabilitiy

$$
\hat{p}_{01}=1, \hat{p}_{10}=0, \hat{p}_{11}=\hat{p}_{12}=\frac{1}{2} \quad \text { and } \quad \hat{p}_{i, i \pm 1}=\frac{1}{2}(i \geqq 2) .
$$

Proof. Since it is known that the time changed process $\hat{X}_{n}$ is a Markov chain, it remains only to check the relations of (10). For this, suppose $X_{0}=1$, then

$$
A_{1}^{-1}=1 \text { iff } X_{1}=2 \text { and } A_{1}^{-1}=2 \text { iff } X_{1}=0 .
$$

By definition of $A_{n}$, this implies

$$
\hat{X}_{1}=X\left(A_{1}^{-1}\right)=X_{1} \text { or } X_{2} \text { according as } X_{1}=2 \text { or } X_{1}=0 \text {, }
$$

so that we have

$$
\begin{aligned}
& P_{1}\left\{\hat{X}_{1}=1\right\}=P_{1}\left\{X_{1}=0, X_{2}=1\right\}=\frac{1}{2}, \\
& P_{1}\left\{\hat{X}_{1}=2\right\}=P_{1}\left\{X_{1}=2\right\}=\frac{1}{2} .
\end{aligned}
$$

The rests of (10) are obvious.

THEOREM 1. Let $\left[S_{n} ; n \geqq 0\right]$ be the standard random walk on all integers $Z$. Then we can construct a new standard random walk from the square process $\left[S_{n}^{2} ; n \geqq 0\right]$.

3) $\chi_{N}$ denotes the indicator function of the set $N=\{1,2,3, \cdots\}$ 
Proof. Let $X_{n}$ be the root process of $S_{n}^{2}: X_{n}=\sqrt{S_{n}^{2}}=\left|S_{n}\right|$. Then $\left[X_{n} ; n \geqq 0\right]$ is a Markov chain with the transition matrix (8). So, by lemma 2 , the time changed process $\hat{X}_{n}$ of $X_{n}$ by the additive functional $A_{n}$ of (9) is a Markov chain on $\{0,1,2,3, \cdots\}$ with the transition matrix (10). Because of $\hat{p}_{01}=1, \hat{p}_{10}=0$, we can apply lemma 1 to $\left[\hat{X}_{n}-1 ; n \geqq 0\right]$ to get the new standard random walk

$$
\hat{S}_{n}=\sum_{1}^{n} u\left(\hat{X}_{k}-\hat{X}_{k-1}\right) .
$$

This proves the theorem.

\section{Brownian motion.}

THEOREM 2. Let $\left[B_{t} ; t \geqq 0\right]$ be the standard Brownian motion on real line $\boldsymbol{R}$. Then we can construct a new standard Brownian motion from the square process $\left[B_{t}^{2} ; t \geqq 0\right]$.

In fact, it is known that the root $X_{t}$ of $B_{t}^{2}$ is the reflecting Brownian motion on $[0, \infty)$ and the local time $T_{t}$ of $X_{t}$ at $x=0$ exists:

$$
T_{t}=\lim _{\varepsilon \downarrow 0} \frac{1}{2 \varepsilon} \text { meas }\left\{s \mid s<t, X_{s}<\varepsilon\right\} .
$$

So, if we set

$$
\hat{B}_{t}=X_{t}-T_{t}
$$

then it will be seen that $\left[\hat{B}_{t} ; P_{a}\right](a \geq 0)$ is a new standard Brownian motion starting from $a$.

To prove Theorem 2, suppose we are given a Brownian motion $\hat{B}_{t}$. Then it is known that, considering (12) as a stochastic equation-the Skorohod's equation-with unknown $X_{t}$ and $T_{t}$, it has the unique nonanticipating solution $X_{t}$, the reflecting barrier Brownian motion and $T_{t}$, the local time of $X_{t}$ (see [5], [7]). Therefore, Theorem 2 is easily derived by virtue of the above results.

Finally, we shall give a direct proof of Theorem 2 applying a theorem of Doob ([3], Theorem 11.9). The proof will be given in several steps as follows.

$1^{\circ}$. As the first step, bring the well known formula ([5])

$$
\begin{aligned}
& P_{0}\left\{X_{t} \varepsilon d x, T_{t} \varepsilon d y\right\} \\
& \quad=\left(\frac{2}{\pi t^{3}}\right)^{1 / 2} \exp \left\{-(x+y)^{2} / 2 t\right\}(x+y) d x d y, x, y>0,
\end{aligned}
$$


to obtain

$$
\begin{gathered}
P_{0}\left\{\hat{B}_{t} \varepsilon d a\right\}=\left(\frac{1}{2 \pi t}\right)^{1 / 2} \exp \left\{-a^{2} / 2 t\right\} d a, \quad a \in R, \\
P_{0}\left\{T_{t} \varepsilon d y\right\}=\left(\frac{2}{\pi t}\right)^{1 / 2} \exp \left\{-y^{2} / 2 t\right\} d y, \quad y>0, \\
\left\{\begin{array}{l}
E_{a}\left(X_{t}\right)=\left(\frac{1}{2 \pi t}\right)^{1 / 2}\left[2 t \exp \left(-a^{2} / 2 t\right)+2 a \int_{0}^{a} \exp \left(-u^{2} / 2 t\right) d u\right], \\
E_{a}\left(X_{t}^{2}\right)=a^{2}+t .
\end{array}\right.
\end{gathered}
$$

Moreover, we need the followings;

$$
\left\{\begin{array}{l}
E_{a}\left(e^{-p \sigma}\right)=e^{-\sqrt{2 p} a} \quad a>0, \sigma=\operatorname{Min}\left\{t \mid X_{t}=0\right\}, \\
P_{a}\{\sigma \varepsilon d t\}=\left(\frac{a^{2}}{2 \pi t^{3}}\right)^{1 / 2} \exp \left\{-a^{2} / 2 t\right\} d t .
\end{array}\right.
$$

$2^{\circ}$. With these formulas, we shall show that

$$
\begin{gathered}
E_{a}\left(\hat{B}_{t}\right)=a, \quad a>0, \\
E_{a}\left(\hat{B}_{t}^{2}\right)=a^{2}+t, \quad a>0,
\end{gathered}
$$

(20) $\left[\hat{B}_{t}, \boldsymbol{F}_{t}, P_{a}\right]$ is a martingale with $E_{a}\left\{\left(\hat{B}_{t}-\hat{B}_{s}\right)^{2} \mid \boldsymbol{F}_{s}\right\}=t-s, \quad t>s$, where $F_{t}=\sigma\left\{X_{s} \mid s \leqq t\right\}$ denotes the $\sigma$-algebra generated by $X_{s}, s \leqq t$. Proof of (18). Since

$$
T_{t}(w)= \begin{cases}0 & , \quad t \leq \sigma(w)^{4)} \\ T_{t-\sigma(\omega)}\left(\theta_{o} w\right), & t \geq \sigma(w)\end{cases}
$$

and

$$
P_{a}\left\{\sigma \varepsilon d s, T_{t-s} \varepsilon d y\right\}=P_{a}\{\sigma \varepsilon d s\} P_{0}\left\{T_{t-s} \varepsilon d y\right\}
$$

we see that

$$
\begin{aligned}
E_{a}\left(\hat{B}_{t}\right) & =E_{a}\left(X_{t}-T_{t}\right)=E_{a}\left(X_{t}\right)-E_{a}\left(T_{t} ; t>\sigma\right) \\
& =E_{a}\left(X_{t}\right)-\int_{0}^{t} E_{0}\left(T_{t-s}\right) P_{0}(\sigma \varepsilon d s) \\
\int_{0}^{\infty} e^{-p t} E_{a}\left(\hat{B}_{t}\right) d t & =\int_{0}^{\infty} e^{-p t} E_{a}\left(X_{t}\right) d t-\int_{0}^{\infty} e^{-p t} E_{0}\left(T_{t}\right) d t \int_{0}^{\infty} e^{-p t} P_{0}\{\sigma \varepsilon d s\}
\end{aligned}
$$

So, by the formulas in step $1^{\circ}$, the right hand side becomes

4) $\theta_{t}$ denotes the shift operator on the basic space $W=\{w\}$. 


$$
\begin{aligned}
\frac{a}{p}(1 & \left.+\frac{1}{\sqrt{2 p} a}\right) e^{-\sqrt{2 p} a}+\frac{a}{p}\left(1-e^{-\sqrt{2 p} a}\right)-\frac{1}{\sqrt{2 p^{3}}} e^{-\sqrt{2 p} a} \\
& =a / p
\end{aligned}
$$

Consequently, we get $E_{a}\left(\hat{B}_{t}\right)=a$.

Proof of (19). Observe that

$$
\begin{aligned}
& \int_{0}^{\infty} e^{-p t} E_{a}\left(X_{t} T_{t}\right) d t=\int_{0}^{\infty} e^{-p t} E_{a}\left(X_{t} T_{t} ; t>\sigma\right) d t \\
& =\int_{0}^{\infty} e^{-p t} d t E_{a}\left(X_{t-\sigma}\left(\theta_{\sigma} w\right) T_{t-\sigma}\left(\theta_{o} w\right) ; t>\sigma\right) d t \\
& =\int_{0}^{\infty} e^{-p t} d t\left[\iint_{x, y>0} x y \int_{0}^{t} P_{0}\left\{X_{t-s} \varepsilon d x, T_{t-s} \varepsilon d y\right\} P_{a}\{\sigma \varepsilon d s\}\right] \\
& =\int_{0}^{\infty} e^{-p t} d t\left[\iint_{x, y>0} x y(x+y) d x d y \int_{0}^{t} \frac{a}{\sqrt{2 \pi s^{3}}} e^{-a^{2} / 2 s^{2}} \frac{2}{\sqrt{2 \pi(t-s)^{3}}}\right. \\
& \left.\quad \times e^{-(x+y) 2 / 2(t-s)} d s\right] \\
& =2 e^{-\sqrt{2 p} a} \iint_{x, y>0} x y e^{-\sqrt{2 p}(x+y)} d x d y \\
& =e^{-\sqrt{2 p} a} / 2 p^{2},
\end{aligned}
$$

and

$$
\int_{0}^{\infty} e^{-p t} E_{a}\left(T_{t}^{2}\right) d t=e^{-\sqrt{2 p} a} / p^{2}
$$

imply

$$
\begin{gathered}
\int_{0}^{\infty} e^{-p t} E_{a}\left(\hat{B}_{t}^{2}\right) d t=\int_{0}^{\infty} e^{-p t}\left[E_{a}\left(X_{t}^{2}\right)-2 E_{a}\left(X_{t} T_{t}\right)+E_{a}\left(T_{t}^{2}\right)\right] d t \\
=a^{2} / p+1 / p-2 e^{-\sqrt{2 p} a} / 2 p^{2}+e^{-\sqrt{2 p} a} / p^{2} \\
=a^{2} / p+1 / p
\end{gathered}
$$

so that $E_{a}\left(\hat{B}_{t}^{2}\right)=a^{2}+t$.

Proof of (20). Noting (18), we get

$$
\begin{aligned}
E_{a}\left[\hat{B}_{t+s} \mid \boldsymbol{F}_{s}\right] & =E_{a}\left[X_{t+s}-T_{t+s} \mid \boldsymbol{F}_{s}\right] \\
& =E_{a}\left[X_{t}\left(\theta_{s} w\right)-T_{s}(w)-T_{t}\left(\theta_{s} w\right) \mid \boldsymbol{F}_{s}\right] \\
& =E_{X_{s}}\left[X_{t}-T_{t}\right]-T_{s}=E_{X_{s}}\left(\hat{B}_{t}\right)-T_{s} \\
& =X_{s}-T_{s}=\hat{B}_{s} .
\end{aligned}
$$

Thus we know that $\hat{B}_{t}$ is a martingale. Next, to show the latter part of (20), it is enough to use (18) and (19) at before the last step in the following computations ; 


$$
\begin{aligned}
& E_{a}\left[\left(\hat{B}_{t+s}-\hat{B}_{s}\right)^{2} \mid \boldsymbol{F}_{s}\right] \\
& \quad=E_{a}\left[\left(\left(X_{t+s}-T_{t+s}\right)-\left(X_{s}-T_{s}\right)\right)^{2} \mid \boldsymbol{F}_{s}\right] \\
& \quad=E_{a}\left[\left(X_{t}\left(\theta_{s} w\right)-T_{t}\left(\theta_{s} w\right)-X_{s}\right)^{2} \mid \boldsymbol{F}_{s}\right] \\
& \quad=E_{X_{s}}\left(X_{t}-T_{t}\right)^{2}-2 X_{s} E_{X_{s}}\left(X_{t}-T_{t}\right)+X_{s}^{2} \\
& \quad=E_{X_{s}}\left(\hat{B}_{t}^{2}\right)-2 X_{s} E_{X_{s}}\left(\hat{B}_{t}\right)+X_{s}^{2} \\
& \quad=X_{s}^{2}+t-2 X_{s}^{2}+X_{s}^{2} \\
& \quad=t .
\end{aligned}
$$

Therefore, $\left[\hat{B}_{t}: P_{a}\right](a \geq 0)$ is a Brownian motion starting from $a$ by a Doob's theorem [3]. Since it is easily seen $\left[-\hat{B}_{t}: P_{a}\right](a<0)$ is a Brownian motion with $-\hat{B}_{0}=a$, we conclude the proof of the theorem.

\section{REFERENCES}

[1] R. M. Blumenthal and R. K. Getoor, Markov processes and potential theory, Academic Press, New York, 1968.

[2] H. Cramer and R. M. Leadbetter, Stationary and related stochastic processes, John Wiley and Sons., Inc., New York, 1967.

[ 3 ] J. L. Doob, Stochastic processes, John Wiley and Sons., Inc., New York, 1953.

[4] F. A. Grünbaum, The square of a Gaussian process, Z. Wahr. verw. Geb., 23, 121-124 (1972).

[ 5 ] H. P. Mckean, Stochastic integrals, Academic Press, New York, 1969.

[6] K. Ito and H. P. Mckean, Diffusion processes and their sample paths, Academic Press, New York, 1964.

[7] A. Skorohod, Stochastic equations for diffusion processes in a bounded region 1. 2, Th. Prob. its Appl. 6, 264-274 (1961) ; 7, 3-23 (1962).

Nagoya University 